\title{
To freeze or not to freeze: heating the debate but cooling the practice?
}

\author{
Paolo F. Rinaudo ${ }^{1}$. John Hsu ${ }^{2,3}$
}

Received: 3 January 2017 / Accepted: 5 January 2017 / Published online: 14 January 2017

(C) Springer Science+Business Media New York 2017

The paper by Luke et al. published in this issue of JARG is bound to heat up the debate about freezing embryos (frozen embryo transfer, FET, versus transfer of fresh embryos) during in vitro fertilization. The practice of IVF already has moved towards greater use of FET, in part to reduce complications associated with ovarian hyperstimulation and in part to accommodate pre-implantation genetic screening (PGS).

In theory, transferring embryos "after the fresh cycle" could allow the endometrium to "recover" from stimulation and result in implantation within a more "natural" hormonal milieu, i.e., one without the high levels of estrogen and progesterone used for stimulation. Some IVF clinics have jumped on these theoretical and practical advantages and now advocate using only FET [11].

The evidence base for transfer of frozen rather than fresh embryos, however, is more inchoate. There have been a number of observational studies that indicate FETs, compared with fresh embryo transfers, are less frequently associated with pregnancy complications, such as preterm birth, antepartum hemorrhage, perinatal mortality, and low birth weight [7, 8]. Studies relying on observational data, however, are susceptible to substantial bias if important confounders are not adequately measured or controlled, e.g., the indication for the IVF or parental socio-economic differences.

Paolo F. Rinaudo

rinaudop@obgyn.ucsf.edu

1 Department of Obstetrics, Gynecology and Reproductive Sciences, University of California, San Francisco, CA, USA

2 Mongan Institute, Massachusetts General Hospital, Boston, MA, USA

3 Department of Health Care Policy, Harvard Medical School, Cambridge, MA, USA
There have been several registered randomized studies that soon could provide valuable information and are less susceptible to some of these potential biases because of the random assignment. Among the few trials with published results, there also has been controversy even in outcomes as basic as the implantation. For example, the pregnancy rate after a freezeall policy has increased by as much as $30 \%$ in some studies [2, $4,13]$, but not others [14, 15]. Even data from randomized controlled trials, however, are susceptible to potential bias given uncertainty in whether any given cycle results in a birth and the frequent need for multiple treatments before birth [5]. Moreover, there are very few data on long-term outcomes for either the child or mother.

Recognized disadvantages of freezing embryos include inconvenience to patients because of delaying pregnancy and potentially increased cost. There has been less data on potential clinical disadvantages of FET, though two complications appear to be more common: (1) high birth weights $[8,9]$ and (2) pregnancy-induced hypertension $[4,6,10]$.

The paper of Luke et al. brings new information on the subject by use of a creative design and a novel dataset. The authors analyzed 7795 pairs of singleton births (i.e., children delivered by the same mother) conceived with IVF and resulting from a fresh or frozen embryo transfer and born between 2004 and 2013. Using this within-mother design, the authors controlled for the mother and for infertility status, which are important potential confounders. The study also used the Society for Assisted Reproductive Technology Clinic Outcome Reporting (SART-COR) System database, identified eligible births, matched for embryo stage (blastocyst versus non-blastocyst) and infant gender, and then categorized by embryo state (fresh versus frozen) in the first and second births (four groups).

The most remarkable finding is that children born from FET were more likely to be in the top 10 percentile of weight 
(i.e., large for gestational age or LGA, both in absolute weight and weight-for-gestation), than their siblings born from fresh cycles were. This is an important finding, as LGA is associated with complications for the mother (e.g., shoulder dystocia, increase use of Cesarean section, and post-partum hemorrhage) and child (e.g., hypoglycemia, longer hospitalization, and obesity later in life) [1, 17]. The findings, while more exploratory than conclusive, are thought provoking and overall are consistent with the belief that both the embryo and mother are sensitive to hormonal or embryonic manipulation [3].

Obviously, more work is needed to confirm and explain the results. For example, there are at least two potential mechanisms by which FET might lead to heavier newborns. First, the endometrium following a FET might still not be a "normal" physiologic endometrium because of use of hormones (albeit at low dose). Second, the freezingthawing process itself could influence the embryo [16] and its related embryonic development, e.g., induce a reprogramming of the fetus to gain weight more rapidly. Future studies with better data will help clarify the underlying pathophysiology. For example, studies that account for exogenous hormone use (i.e., "medicated" FET) versus not (e.g., "natural" FET), and for the factors leading to the decision to administer the hormones, could shed light on the mechanism for the observed weight gain.

The study design also has a number of important limitations associated with using observational data and with the limitations of the dataset. For example, the study did not include number of important potential confounders, e.g., parental socio-economic status, weights, dietary or exercise behaviors, alcohol or drug use, or prenatal care. The within-mother design protects somewhat against the missing data, but these characteristics might influence the likelihood that many women even enter the dataset (i.e., have multiple children through IVF) or interact with the treatment.

Even the nature of the infertility and the potential relationship between infertility and the choice of treatments is largely unknown in this dataset. The longer-term impact of these treatments on the health of the child and mother are paramount considerations, but outside the scope of this study. The authors readily acknowledge the limitations of their dataset. In future studies, use of richer, longitudinal datasets and more sophisticated methods of analysis will help addressing the potential confounders.

Finally, the study's use of the SART-COR database also is laudable. Much of medicine is moving towards use of all existing data to monitor and study patient outcomes. Arguably, it is time that such big data approaches in examining IVF therapies and for the field to systematically track both clinical innovations and their effects.

In summary, the goal of any IVF program is the birth of a healthy child; thus, these results should give us some pause in the rush to FET. Until we have more evidence on the ramifications of freezing embryos, IVF clinicians might want to consider the potential trade-offs in each case, i.e., an individualized policy. For example, while it is clear that high levels of estrogens or progesterone have deleterious effects on the endometrium with subsequent lower implantation and pregnancy rate, the amount of stimulation and treatment history vary across cases. In some cases involving less stimulation or for mothers with lower ovarian reserve, a fresh transfer might very well be preferred [12]. As future studies confirm or dispel these findings, physicians should use sound judgment before adopting FET as a panacea for all patients.

\section{References}

1. Baird J, Fisher D, Lucas P, Kleijnen J, Roberts H, Law C. Being big or growing fast: systematic review of size and growth in infancy and later obesity. BMJ. 2005;331:929.

2. Blockeel C, Drakopoulos P, Santos-Ribeiro S, Polyzos NP, Tournaye H. A fresh look at the freeze-all protocol: a SWOT analysis. Hum Reprod. 2016;31:491-7.

3. Bloise, E, SK Feuer, and PF Rinaudo. Comparative intrauterine development and placental function of ART concepti: implications for human reproductive medicine and animal breeding. Hum Reprod Update. 2014.

4. Chen ZJ, Shi Y, Sun Y, Zhang B, Liang X, Cao Y, et al. Fresh versus frozen embryos for infertility in the polycystic ovary syndrome. $\mathrm{N}$ Engl J Med. 2016;375:523-33.

5. Hernan MA, Hernandez-Diaz S, Robins JM. Randomized trials analyzed as observational studies. Ann Intern Med. 2013;159: $560-2$.

6. Luke B, Stern JE, Kotelchuck M, Declercq ER, Anderka M, Diop $\mathrm{H}$. Birth outcomes by infertility treatment: analyses of the population-based cohort: Massachusetts Outcomes Study of Assisted Reproductive Technologies (MOSART). J Reprod Med. 2016;61:114-27.

7. Maheshwari A, Pandey S, Shetty A, Hamilton M, Bhattacharya S. Obstetric and perinatal outcomes in singleton pregnancies resulting from the transfer of frozen thawed versus fresh embryos generated through in vitro fertilization treatment: a systematic review and meta-analysis. Fertil Steril. 2012;98(368-377):e361-369.

8. Maheshwari, A, EA Raja, and S Bhattacharya. Obstetric and perinatal outcomes after either fresh or thawed frozen embryo transfer: an analysis of 112,432 singleton pregnancies recorded in the Human Fertilisation and Embryology Authority anonymized dataset. Fertil Steril. 2016.

9. Nakashima A, Araki R, Tani H, Ishihara O, Kuwahara A, Irahara M, et al. Implications of assisted reproductive technologies on term singleton birth weight: an analysis of 25,777 children in the national assisted reproduction registry of Japan. Fertil Steril. 2013;99:450-5.

10. Opdahl S, Henningsen AA, Tiitinen A, Bergh C, Pinborg A, Romundstad PR, et al. Risk of hypertensive disorders in pregnancies following assisted reproductive technology: a cohort study from the CoNARTaS group. Hum Reprod. 2015;30:1724-31.

11. Roque M, Valle M, Guimaraes F, Sampaio M, Geber S. Freeze-all policy: fresh vs. frozen-thawed embryo transfer. Fertil Steril. 2015;103:1190-3.

12. Roque, M, M Valle, F Guimaraes, M Sampaio, and S Geber. Freeze-all cycle for all normal responders? J Assist Reprod Genet. 2016. 
13. Shapiro BS, Daneshmand ST, Garner FC, Aguirre M, Hudson C, Thomas S. Evidence of impaired endometrial receptivity after ovarian stimulation for in vitro fertilization: a prospective randomized trial comparing fresh and frozen-thawed embryo transfer in normal responders. Fertil Steril. 2011;96:344-8.

14. Shapiro BS, Daneshmand ST, Garner FC, Aguirre M, Hudson C, Thomas S. Evidence of impaired endometrial receptivity after ovarian stimulation for in vitro fertilization: a prospective randomized trial comparing fresh and frozen-thawed embryo transfers in high responders. Fertil Steril. 2011;96:516-8.
15. Vuong LT, DV, Ho TM, Huynh BG, Ha DT, Pham TD, Nguyen LK, Norman RJ, Mol BW. Freeze-all versus fresh embryo transfer in IVF/ICSI, a randomised controlled trial (NCT02471573). p. O269: ASRM 2016 annual conference. 2016.

16. Wang L, Zhang X, Yu C, Yao Y. Gene expression profiles of in vivo derived mouse blastocysts after slow-freezing. Front Biosci (Elite Ed). 2011;3:1162-8.

17. Weissmann-Brenner A, Simchen MJ, Zilberberg E, Kalter A, Weisz B, Achiron R, et al. Maternal and neonatal outcomes of large for gestational age pregnancies. Acta Obstet Gynecol Scand. 2012;91:844-9. 